\title{
Liowsn Project: An Operating System Remastered for Works with Simulation of Wireless Sensor Networks
}

\author{
Marllus de MeloLustosa \\ Federal University of Piauí \\ UFPI - Brazil \\ Teresina - PI
}

\author{
Shivjay Singh \\ Research Scholar \\ School of Study-Computer Science \\ Jiwaji University - Gwalior
}

\section{ABSTRACT}

Since the last decade, the area of Wireless Sensor Networks (WSN) is a field search on the rise. Due to its high mobility and scalability in several areas, acting on functions of sensing, monitoring, tracking, control and maintenance, WSNs are increasingly attracting researchers for the study. With the advent of computer simulations, it has been eliminated, in part by the necessity of obtaining real sensors, which because of its implementation cost, eventually disabling the initial analysis and further tests with these networks. Considering that most of these simulator programs are native from GNU/Linux, expertises are needed to implement these tools in the operating system, which can be a problem. This paper presents the Liowsn project, which is a GNU/Linux operating system remastered with several classic tools of literature already installed and configured, and it has been proposed in order to achieve a higher speed in the implementation process of the simulation environment to study this type of network.
\end{abstract}

\section{Keywords}

Wireless Sensor Networks, WSNs, Networks, Simulation, Liowsn

\section{INTRODUCTION}

The Wireless Sensor Networks (WSN) are formed by a large number of mobile and small sensors, called node sensors, which are distributed within a given area or base to detect and transmit the physical characteristics of an environment. In general, WSN can be used in security, monitoring, control, operation and maintenance of complex systems, and monitoring of internal and external environments [1]. Among the main components of a Wireless Sensor Network, you can quote: the sensor, the observer and the phenomenon. Figure 1, it shows the basic operation of this type of environment.

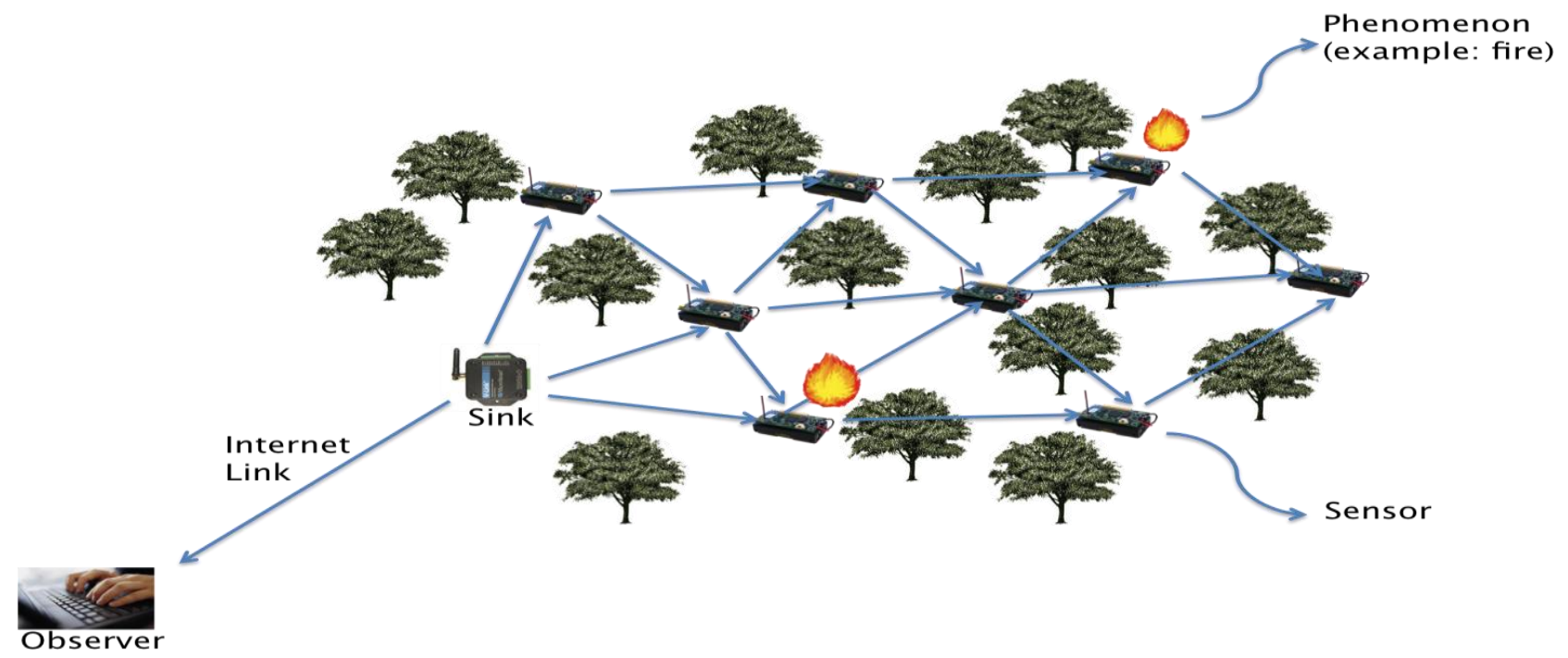

Fig 1: Basic example of a WSN.

Through the wide range of available problems not studied or that are still in the initial study, the researches on WSN only tend to grow. Therefore, it is very important the use of computational simulations to aid researchers in this study, besides competing with other relevant highly factors, which are:

- High cost to assemble a real WSN;
- $\quad$ Electronic components of WSN with difficult access and use with little documentation;

- Simulation possibility of several types of real environments, typical of WSN;

Several tools are used for the studies in this area. Some of them have special prominence in the literature such as simulators NS-2, Castalia, OMNeT++ reports and analyzers (trace files generated by the simulators), and Tracegraph as 
xgraph. However, a great deal of time to create an operating environment for simulation of WSN is still a problem. The lack of participation of researchers in discussion groups of simulators, the difficulty of installing these tools in the operating system as in [2], the library dependencies and/or other tools that ordering, coupled with the lack of updating, for most of those who develop them, which the main factors that contribute to the motivation of those involved in projects in the area.

This paper presents the Liowsn project (for Linux works with wsn), developed with the aim of solving part of the great difficulty in research on networks by the researches, especially sensor networks, in the process of implementing the necessary environment to computational simulation in this area.

\section{COMPUTER SIMULATION}

In computing, the simulation term is to employ formalizations on computers, such as mathematical expressions or specifications more or less formalized, with the purpose of representing a process or operation of the real world. Thus, in order to be performed a simulation, it is necessary to build a computer model that matches the real situation that you want to simulate. [3]

The computer simulation of systems is the use of mathematical techniques used on computers, which allow you to "mimic" the operation of virtually any type of operation or real-world process; it means that is the study of the behavior of real systems through the exercise of models. [3] [4]

\section{THE STUDY OF WSN}

There are several tools that perform the simulation of real environments by collecting data in a WSN, such as Network Simulator (NS-2) and Java in Simulation Time (JIST). These tools allow the configuration of a common network or a WSN through the parameters such as size of the simulation area, the amount of used sensor nodes and the location of sensor nodes, layer application of the network, layer access to middle of the network, network routing protocol, potency of antenna of the sensor nodes, amount of available energy in each sensor node, consumption for sending and receiving data from sensor nodes, among others. After the simulation, these tools generate files called trace files, containing data that describes which events occurred in the simulated network and what moment in time. [5]

Several features can be analyzed through these trace files, such as:

- Average rate of packets sent and received by the sensor nodes;

- The energy consumed by the network at a given instant of time;

- Lifetime of sensor nodes;

Study Wireless Sensor Network is not a trivial task, especially with regard to the process of validation and testing of new protocols and architectures. The simulation in this type of study is essential to the success of the research, which wins superiority against the practice tests, since this method becomes almost impossible, by high costs in obtaining sensors, in addition to other factors already mentioned in this article.

\section{THE LIOWSN PROJECT}

Deploy the computational environment required for initiation to research in the area of Wireless Sensor Network is the first step to the researcher who wants to perform simulations throughout your study. In the literature, some classic tools such as NS-2 and OMNeT++ still hold the preference of the vast majority of researchers who begin their work. However, in addition to installing these tools many times it is necessary the configuration of additional modules for specific studies with Wireless Sensor Network, since that these simulators work with networks in general. As new modules, the researcher should rebuild the Simulator loaded with the new packages. Considering that the vast majority of these programs are native to the Linux platform, it is natural to have a technical body suitable for proper configuration of the operating system, which, seeing the big list of questions about installation as in [2], as well as doubts about adding new modules in large forums of these programs as in [2] [6] [7] [8], it is understood that this task is not trivial.

From this scenario, the Liowsn Project was proposed consisting of a GNU/Linux operating system, remastered with the entire introduction to research in the area of Wireless Sensor Network created and configured. Operating system were added in several classical tools for simulation of Wireless Sensor Network, among them the NS-2 and $\mathrm{OMNeT}++$, in addition to some specific frameworks for work in this area, as the Castalia and Mannasim. The description of all the tools included in the operating system follows in the next topics.

\section{DESCRIPTION OF OPERATION SYSTEM}

The operating system chosen to compose the Liowsn Project was the GNU/Linux Ubuntu 9.10. The tools were installed in the operating system and available for use without the need for extra configuration or installation of modules. All are described below.

\subsection{NS-2.34 (Network Simulator)}

Ns-2 is a simulator of discrete network in event-oriented research. Ns-2 provides substantial support for simulation of $\mathrm{TCP}$, routing and multicast protocols in wired and wireless (local and satellite). Ns-2 is scribed in $\mathrm{C}++$ and object oriented version of Tcl called OTcl. [9] [10]. It is highly scalable with characteristics of coupling modules for simulating different types of networks. In the installing the NS-2.34 is still followed the NAM (Network Animator), which is a tool capable of performing a $2 \mathrm{D}$ animation of the simulation.

\subsection{Mannasim}

The Mannasim framework is a module for simulation of Wireless Sensor Networks based on Network Simulator (NS2). The Mannasim extends NS-2 in the introduction of new modules for the design, development and analysis of different applications of WSN. [11] [12]

It provides a SGT (Script Generator Tool), which has the function of being a front-end for easy creation of scripts TCL simulation. The GTS comes with the Mannasim framework and is written in Java. 


\subsection{OMNeT++}

$\mathrm{OMNeT}++$ environment is a discrete event simulation. His primary area of application is the simulation of communication networks, but because of its generic and flexible architecture, is successfully used in other areas like the simulation of complex IT systems, queuing networks as well as hardware architectures. [13]

$\mathrm{OMNeT}++$ provides a architecture of components for models. Components (modules) are programmed in $\mathrm{C}++$, and then assembled into components and models larger using a high level language (NED). Reuse of models comes for free. $\mathrm{OMNeT}++$ has extensive GUI support, and due to its modular architecture the core simulation (and models) can be easily embedded into your applications. [13] [14]

\subsection{Castalia}

Castalia is a simulator for wireless sensor networks (WSN), Body Area Network (BAN) and, generally, networks of embedded devices of low power. It is based in the OMNeT++ platform and can be used by researchers and developers who want to test their distributed algorithms and/or protocols in realistic wireless channel and radio models with a realistic node behavior especially related to a radio access. Castalia can also be used to evaluate the different platforms characteristics for specific applications, and can simulate a wide range of platforms. [15]

\subsection{Xgraph}

Xgraph is a general purpose $\mathrm{x}-\mathrm{y}$ data plotter with interactive buttons for panning, zooming, printing, and selecting display options. It will plot data from any number of files on the same graph and can handle unlimited data-set sizes and any number of data files. [16]

Xgraph is a trace analyzer widely used in the literature. Because it contains many features for viewing and has high scalability, it iskeeped up with the installation files on the NS2.

\subsection{Tracegraph}

Trace graph is a Network Simulator ns trace files analyser. It shows simulation and nodes statistics, plots graphs $(2 \mathrm{D}, 3 \mathrm{D}$, histograms) and it saves all the analysis results. [17]

Although the average popularity of this software, its functionality is powerful because it uses features of Matlab, thus providing a wide range of views and analysis on graphs.

\section{IMAGES OF OPERATION SYSTEM}

Below are images of the operating system GNU/Linux Ubuntu 9.10 in operation, with examples of tools and simulations in functioning.

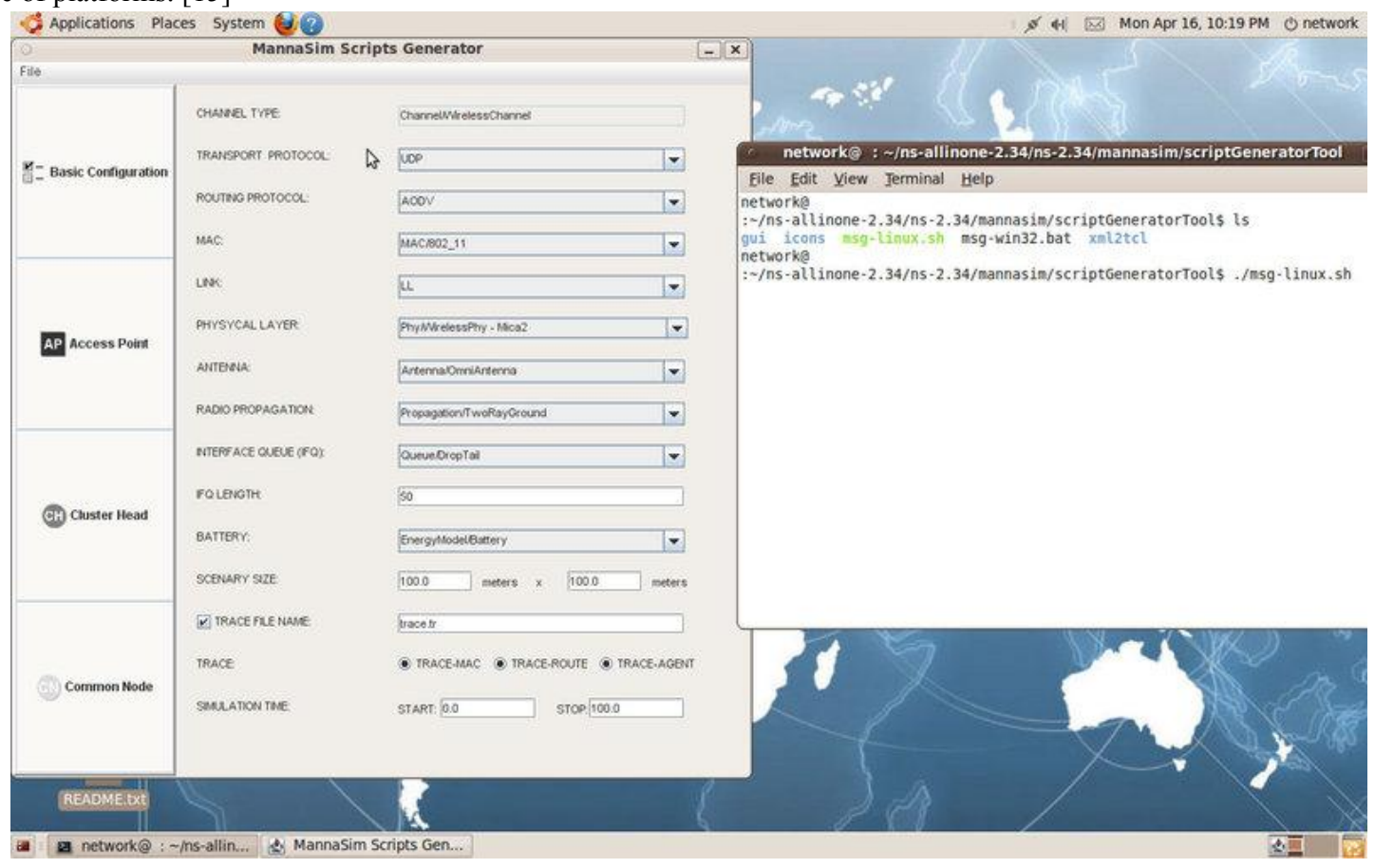

Fig 2: Example of utilization of the Manasim 


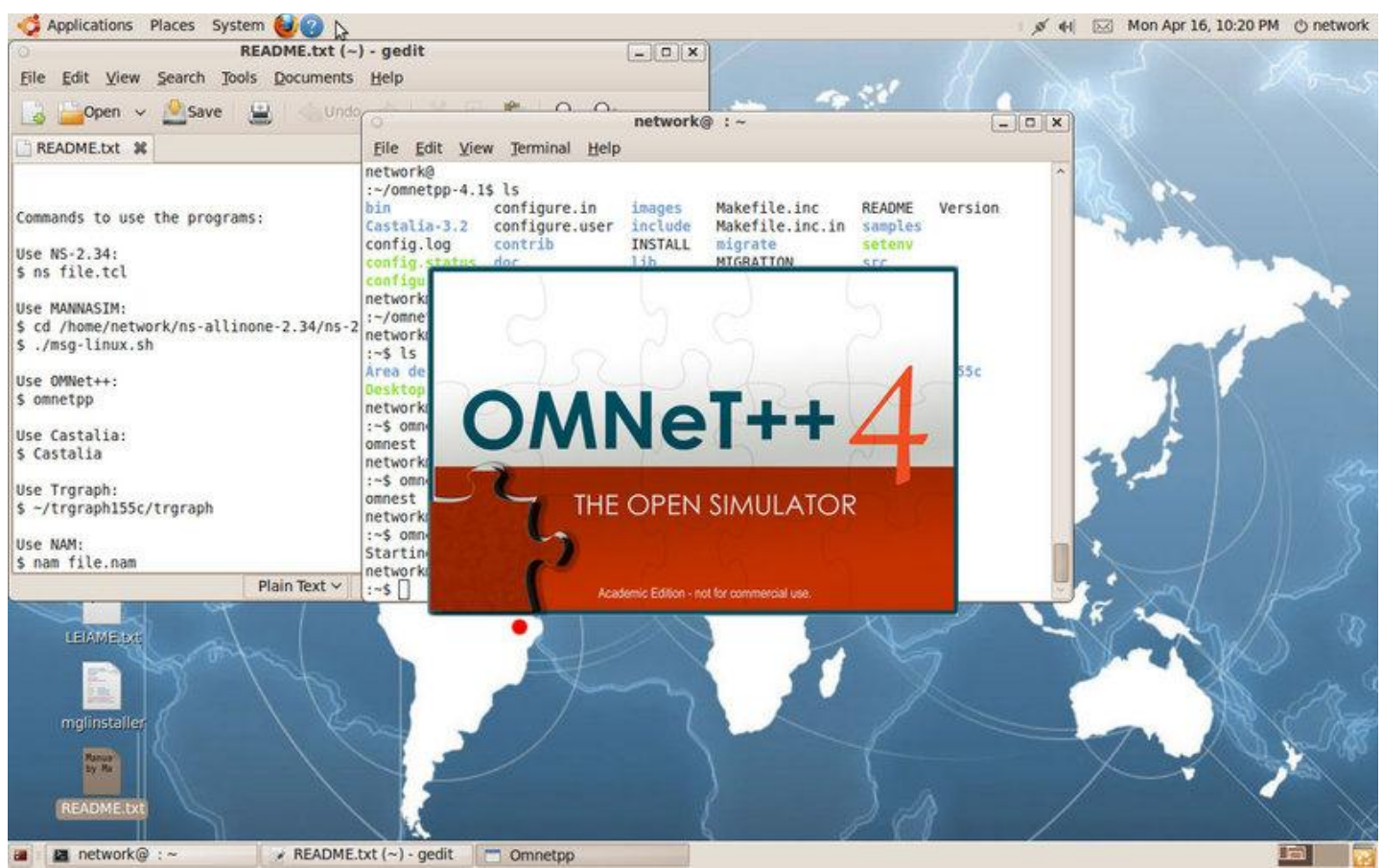

Fig 3: Example of utilization of the OMNeT++



Fig 4: Example of simulation with TCL Script - NS-2.34 + Mannasim 


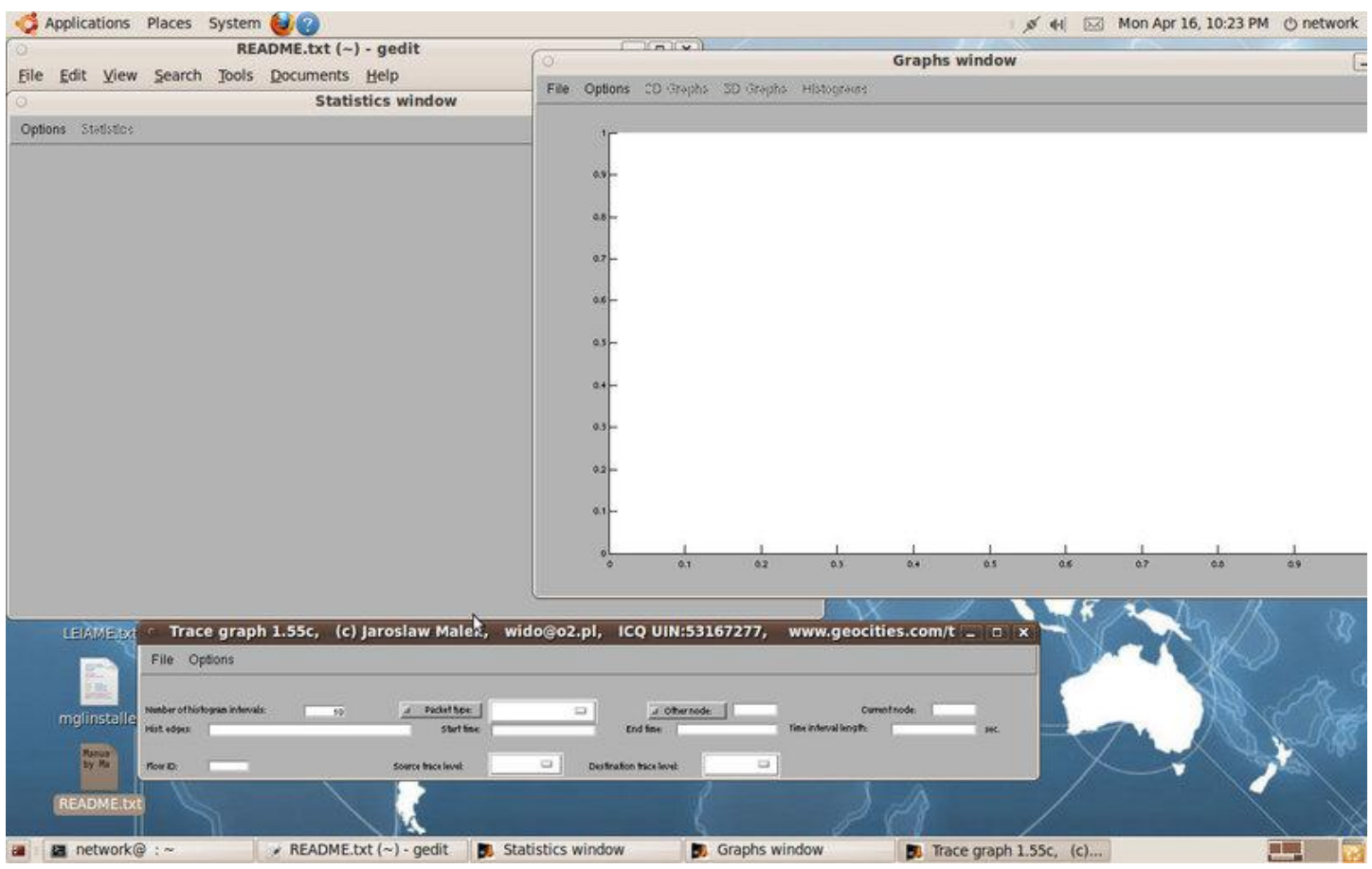

Fig 5: Example of utilization of the Trace graph

\section{USAGE DOCUMENTATION}

For the use of the resources of the Liowsn project was created the site project (http://sourceforge.net/projects/liowsn/), where you will find the download of the operating system, which is currently in version 0.1 . Theremastered operating system GNU/Linux Ubuntu 9.10 comes in its original GNU GPL and can be distributed from the prerogatives of the official documentation. All system constituents tools are under their official licenses and were not changed.

The system's manual which offers steps on how to use the operating environment, as well as handling the tools for simulation of Wireless Sensor Network is available on the official website of the project at the disposal of professionals and academic community. In addition, there is also your discussion group, which constitutes a space for questions and suggestions from users, and can be found at (http://groups.google.com/group/liowsn-users/).

\section{FUTURE WORKS}

For future works, the research group will analyze the Project Liowsn acceptance in the academic community, from the perspective of quantitative and qualitative experienced researchers and beginners professionals /students in the area, and compare with the use of other tools in the literature, observing aspects of deployment time of the simulation environment and the level of difficulty in this process.

The WSN area still has many open issues, such as the problem of power consumption of sensor nodes, as well as improvements in routing protocols in each type of environment, making them work in an increasingly collaborative form, minimizing delay or data loss in case of failure or loss of sensor nodes. The group also intends to research on these problems covering this referred area, using as the simulation environment, the operating system and constituent tools of the Liowsn Project.

\section{CONCLUSION}

Note that research with Wireless Sensor Networks are an area quite comprehensive, as there are still many points open in multiple specificities inserted on this study. And to the researcher or group which you want to study this large area are required in addition to knowledge about their own Wireless Sensor Network protocols and architectures, an entire environment necessary for initiation to research, which includes installed, configured computer simulators and ready for use, along with specific modules for work with Wireless Sensor Networks, which can make the hard work if there is no technical expert in Linux systems in the creation of this simulation environment. The Liowsn project has thus made public the operating system capable of addressing the shortcomings in relation to this problem. This system consists of several discussed and used tools and modules in specific literature, which will reduce much of the time spent in the deployment of the software and will eliminate the requirement for additional technical body that would create all the operating environment for work with these networks! From this, the project aims to encourage the increase in productivity of researchers who aspire to success in theme.

\section{REFERENCES}

[1] Loureiro, A.A.F., Nogueira, J.M.S., Ruiz, L.B., Mini, R.A.F., Nakamura, E.F., Figueiredo, C.M.S. 2003."Redes de sensoressemfio". Brazilian Symposium on computer networks.

[2] Nabble. 2012. Network Simulator ns-2 - The Official Discussion List. Research on errors in the installation of the

ns-

2.http://old.nabble.com/forum/Search.jtp?query=installati on + error + ns \&local $=y \&$ forum $=15582 \&$ daterange $=0 \&$ star tdate $=$ \&enddate $=/$

[3] Freitas, P.J.F. 2008.Introdução à Modelagem e Simulação de Sistemas: comAplicaçõesem Arena. 2. ed. 
Florianópolis: Visual Books Ltda. 372p. ISBN 978-857502-228-3.

[4] Chwif, L., Medina, A.C. 2006.Modelagem e Simulação de EventosDiscretos: Teoria e Aplicações. 1 ed. São Paulo: Bravarte. 254 p. ISBN 85905978-1-4.

[5] Verona, A.B. 2010.Simulação e análise de redes de sensoressemfioaplicadas à viticultura. Dissertation - MSc in computer science. v548s. State University of Maringá - Brazil, Graduate Program in Computer Science.

[6] The Linux Questions. 2012. Forum of the linux users. Research on errors in the installation of the module Mannasim in Ns-2. http://www.linuxquestions.org/questions/search.php?sear chid $=5438840$.

[7] Gname. 2012. Forum of discussion on the NS-2. Research on errors in the installation of the module Mannasim in Ns-2. $\mathrm{http} / / /$ search.gmane.org/?query=mannasim+error+installa tion\&author $=\&$ group $=$ gmane.network.simulator.isi\&sort $=$ relevance $\&$ DEFAULTOP $=$ and $\& \mathrm{xP}=\mathrm{Zmannasim} \% 09 \mathrm{Ze}$ rror\&xFILTERS $=$ Gnetwork.simulator.isi---A

[8] The Mail Archive. 2012. Discussion list of the ns-users. Research on errors in the installation of modules on the ns-2. http://www.mail-archive.com/search?1=nsusers\% $\%$ isi.edu\&q=error+installation+module.

[9] The Network Simulator - ns-2. 2001. The Official Documentation. http://www.isi.edu/nsnam/ns/.
[10] Zhang, J., Li, W., Cui, D., Zhao, X., Yin, Z. 2009. The NS2-Based Simulation and Research on Wireless Sensor Network Route Protocol. WiCom '09. doi: 10.1109/WICOM.2009.5302699

[11] Mannasim. Official page of the Project. http://www.mannasim.dcc.ufmg.br/.

[12] Braga, T.R.M., Silva, F., Ruiz, L.B., Nogueira, J.M.S. 2004. Mannasim: Um arcabouçoparasimulação de Redes de SensoresSemFio. Electronic magazine of scientific Initiation.

[13] Xian, X., Shi, W., Huang, H. 2008. Comparison of OMNET++ and other simulator for WSN simulation. 3rd IEEE Conference on Industrial Electronics and Applications. doi: 10.1109/ICIEA.2008.4582757.

[14] Varga, A.: OMNET++ - Discrete Event Simulation System Version 4.2 User Manual. http://www.omnetpp.org/doc/omnetpp/manual/usman.ht $\mathrm{ml}$.

[15] Castalia - A simulator for Wireless Sensor Networks and Body Area Networks Version 3.2 User's Manual. http://castalia.npc.nicta.com.au/pdfs/Castalia\%20\%20User\%20Manual.pdf.

[16] Xgraph - General Purpose 2-D Plotter. 2012. Official page of the Project. http://www.xgraph.org/.

[17] Malek, J. 2002. Trace Graph Program Download Page. Official page of the Project. http://www.angelfire.com/al4/esorkor/. 